\title{
TERAPIA OCUPACIONAL EN SALUD MENTAL DE CORTA ESTADÍA EN CLÍNICA DE ATENCIÓN PRIVADA
}

\author{
OCCUPATIONAL THERAPY IN SHORT TERM MENTAL HEALTH CARE IN A PRIVATE
}

CLINIC

\section{Andrea Eberhard Aguirre'; Paula Torrico Mayol ${ }^{2}$}

\begin{abstract}
Resumen
Presentamos la experiencia de 12 años atendiendo pacientes que padecen patologías psiquiátricas agudas, en un servicio de corta estadía, inserto en una clínica de atención general, universitaria y privada.

Se diseñó un programa de atención semanal que incluye variadas actividades.

Las intervenciones son desarrolladas por el equipo de terapia ocupacional en una sala dentro del servicio de hospitalización, a la cual se integran los pacientes en cuanto la sintomatología lo permite.

El programa se ajusta a las necesidades e intereses de los usuarios de cada semana, sus características de flexibilidad permite que las actividades con propósito, forma, significado y en un contexto adecuado promuevan la recuperación.

Los pacientes son entrevistados y evaluados al inicio del proceso terapéutico y evolucionados después de las actividades en las que participan, sean individuales o grupales, siendo discutidos entre las TO y también con el equipo tratante.

La psicoeducación familiar y la docencia a estudiantes de TO y medicina es otra parte importante de nuestra rutina laboral.

Un $81 \%$ de los pacientes perciben la ayuda recibida de TO como beneficiosa. Máxima calificación consignada en una tesis doctoral (2012) realizada en la clínica en pacientes con intento de suicidio grave.

Desde el 2001 han ingresado 4.150 pacientes de los cuales sobre el 95\% participan en TO. El 2011 recibimos $47 \%$ trastornos del ánimo, seguido de $18 \%$ adicciones; $9,1 \%$ esquizofrenia; $6 \%$ T. Alimentarios.

La modalidad de trabajo descrita ha demostrado ser posible y muy bien evaluada por los pacientes, familias y alumnos.
\end{abstract}

Palabras clave:

TO en corta estadía, TO en salud mental, Ergoterapia en salud mental, TO y equipo multidisciplinario de salud mental.

1 Terapeuta Ocupacional, licenciada en Ciencias de la Ocupación Humana U. de Chile. Terapeuta Ocupacional de hospitalización Salud Mental, Clínica UC San Carlos de Apoquindo. 7620002 -0 27548874. nini.eberhard@gmail.com

2 Terapeuta Ocupacional, licenciada en Ciencias de la Ocupación Humana U. de Chile. Terapeuta Ocupacional de hospitalización Salud Mental, Clínica UC San Carlos de Apoquindo. 7620002 - 027548874. ptorrico@med.puc.cl 


\begin{abstract}
We presented this nearly 12 year-long experience in treating acute psychiatric patients in mental health services befitting the concept of a short term mental health care in a private general care clinic at university level.

A weekly care program was designed and it includes several activities.

The interventions are carried out by an occupational therapy staff; patients join in as soon as their symptoms allow them.

The program adapts to the needs and interests of the patients each week, so its

flexibility allows us to ensure that the activities have a purpose and meaning,

in a context that enhances recovery.

Patients are interviewed and evaluated at the beginning of the therapeutic process and again after participating in activities, whether individual or in a group.

Their progress is constantly discussed among the occupational therapists and with the rest of the staff involved.

Psychoeducational family and OT and medicine student training is another important part of the daily routine.

$81 \%$ of the patients acknowledge the aid as helpful. Top ranking indicated in a doctoral thesis (2012) carried out in the clinic with strong suicidal attempts patients.

Since 2001, 4,150 patients have been registered, and 95\% are in a occupational therapy program.

In 2011, registered $47 \%$ are diagnosed with mood disorders, followed by $18 \%$ substance abuse $9.1 \%$ schizophrenia; $6 \%$ eating disorders.

The working mode described above has proven possible and very well rated by patients, families and students
\end{abstract}

Keywords:

OT in short term care, OT in mental health, hand and craft in mental health; OT in a multidisciplinary mental health team.

\section{INTRODUCCIÓN}

El servicio de Salud Mental de la clínica UC San Carlos de Apoquindo (CSC) se inserta en un centro de atención integral de salud. La hospitalización psiquiátrica de este servicio, fue diseñada y construida especialmente para la atención de pacientes con este tipo de patologías. Esto significa que el paciente cuenta con todo el apoyo propio de una clínica general, con la presencia permanente de un médico psiquiatra, y con infraestructura especialmente diseñada para su cuidado y recuperación.

La hospitalización siquiátrica fue creada para dar atención de excelencia a hombres y mujeres, desde los 15 años de edad, con trastornos mentales en su etapa aguda (promedio 2 a 4 semanas de permanencia), con el fin de establecer un diagnóstico e instaurar un tratamiento; o bien, para superar una crisis actual.

Desde su inicio (2001) el servicio de Salud Mental de la CSC cuenta con la unidad de Terapia Ocupacional (TO).

La TO originalmente fue relacionada solamente con la rehabilitación, los avances profesionales le han permitido desarrollar intervenciones también en la atención secundaria, es decir, atenciones orientadas al tratamiento en crisis, recuperación de funcionalidad y desarrollo de las capacidades del sujeto. La TO ejerce acciones graduadas y sistematizadas, colabora en el diagnóstico y efectúa el tratamiento mediante métodos y técnicas adecuadas con el fin de recuperar psíquica, y funcionalmente al paciente.

Dentro de las estrategias que podemos encontrar en esta unidad:

- Brinda asistencia profesional al paciente.

- Orienta al grupo familiar.

- Participa de un grupo interdisciplinario mediante la evaluación, tratamiento, controles y altas.

La relevancia de esta unidad radica en una doble función, la primera es de diagnóstico, la cual se realiza mediante entrevistas, evaluación y observación clínica del paciente; y la función terapéutica que es la intervención de TO propiamente tal.

El tratamiento consiste en restablecer las funciones disminuidas; psíquicas y sociales, utilizando técnicas y métodos apropiados de acuerdo al trastorno o disfunción que se observe en la persona, con el objetivo de desarrollar las capacidades potenciales y utilizando las habilidades remanentes. (Martijena, N. 1999). 


\section{Experiencia Profesional}

Este trabajo de sistematización, da cuenta de la experiencia que deriva de la práctica asistencial. La misma procura analizar detalladamente la experiencia de un equipo de TO atendiendo pacientes psiquiátricos hospitalizados en una clínica de corta estadía.

Las intervenciones de las terapeutas ocupacionales de esta unidad permiten la observación de los pacientes en un contexto diferente a las aproximaciones terapéuticas del resto del equipo tratante, por lo cual se vuelve fundamental su activa participación en las reuniones clínicas del equipo multidisciplinario que se realizan 3 veces por semana en donde se discute el tratamiento y evolución de cada paciente

El servicio tiene capacidad para 20 camas y la unidad de TO funciona de lunes a viernes en jornada com- pleta y sábado medio día, actualmente cuenta con 3 terapeutas ocupacionales en diferentes jornadas.

Los pacientes pasan la mayor parte del tiempo en la unidad de TO en una rutina de atención que se ha establecido para ellos.

El trabajo de TO se enmarca en diferentes intervenciones según las necesidades de los pacientes y objetivos del equipo multidisciplinario.

La semana se planifica y estructura en torno a un tema de trabajo organizado en ciclos. Dentro de esta estructura el día se divide en dos instancias fundamentales que tienen objetivos diferentes, durante la mañana se desarrolla el tema de la semana en las actividades grupales, intervenciones individuales, y durante la tarde se desarrolla Ergoterapia.

En el siguiente cuadro, se expone la organización semanal de las intervenciones por parte de T.O.

\begin{tabular}{|c|c|c|c|c|c|}
\hline Lunes & Martes & Miércoles & Jueves & Viernes & Sábado \\
\hline $\begin{array}{l}\text { Reunión Clínica } \\
\text { Evaluación de permi- } \\
\text { sos terapéuticos, } \\
\text { presentación de } \\
\text { ingresos al grupo } \\
\text { Presentación Tema } \\
\text { semanal }\end{array}$ & Relajación & $\begin{array}{l}\text { Reunión Clínica } \\
\text { Planificación de la } \\
\text { semana del equi- } \\
\text { po de TO. } \\
\text { Presentación de } \\
\text { seminario de in- } \\
\text { terno de T.O. } \\
\text { Grupo de Psico- }\end{array}$ & $\begin{array}{l}\text { Dinámica } \\
\text { Grupal }\end{array}$ & $\begin{array}{l}\text { Reunión clínica } \\
\text { Estimulación } \\
\text { cognitiva o } \\
\text { sensorial } \\
\text { Preparación de } \\
\text { permisos tera- } \\
\text { péuticos de fin } \\
\text { de semana. }\end{array}$ & $\begin{array}{c}\text { Relajación y/o act. } \\
\text { Física. } \\
\text { Dinámica grupal } \\
\text { Revisión de cumpli- } \\
\text { miento de metas de } \\
\text { la semana y cierre } \\
\text { del tema } \\
\text { Ergoterapia }\end{array}$ \\
\hline $\begin{array}{c}\text { Elaboración de metas } \\
\text { personales según el } \\
\text { tema de la semana }\end{array}$ & terapéutica & $\begin{array}{c}\text { terapia } \\
\text { Actividad física }\end{array}$ & & $\begin{array}{l}\text { Plan de alta } \\
\text { Actividad física }\end{array}$ & \\
\hline Almuerzo & Almuerzo & Almuerzo & Almuerzo & Almuerzo & \multirow{3}{*}{ Almuerzo } \\
\hline $\begin{array}{c}\text { Ergoterapia/ } \\
\text {-Uso PC }\end{array}$ & $\begin{array}{c}\text { Ergoterapia } \\
\text {-Uso PC }\end{array}$ & $\begin{array}{c}\text { Ergoterapia } \\
\text {-Uso PC }\end{array}$ & $\begin{array}{c}\text { Ergoterapia } \\
\text {-Uso PC }\end{array}$ & $\begin{array}{c}\text { Ergoterapia } \\
\text {-Uso PC }\end{array}$ & \\
\hline Evolucionar fichas & Evolucionar fichas & Evolucionar fichas & $\begin{array}{c}\text { Evolucionar } \\
\text { fichas }\end{array}$ & $\begin{array}{c}\text { Evolucionar } \\
\text { fichas }\end{array}$ & \\
\hline
\end{tabular}


Las intervenciones del equipo de TO de CSC se clasifican en:

\section{Trabajo grupal}

Los pacientes participan en actividades grupales orientadas a brindar un espacio social que invite a la reflexión e introspección. El trabajo grupal se establece según el tema de la semana organizado en 5 ciclos de trabajo:

- Manejo de las emociones

- Habilidades sociales

- Autocuidado

\section{- Autoestima}

- Bienestar subjetivo y calidad de vida

Los ciclos de trabajo se eligen de acuerdo a la necesidad general de los pacientes.

El compartir experiencias con pares en situación similar, beneficia la discusión para entender de mejor manera las diferentes problemáticas que aquejan a los pacientes y los llevan a la situación de enfermedad. Por otro lado permite un espacio de autoayuda, de soporte y distensión del estrés que significa la hospitalización.

Para el equipo de TO, es además una instancia de evaluación de habilidades y desenvolvimiento social.

Los trabajos grupales que se realizan en el servicio de salud mental de CSC, se organizan como lo señala el siguiente cuadro:

\subsection{Dinámicas grupales}

Que buscan cumplir con el objetivo del tema de la semana según el ciclo. Es a través de la participación, exposición y discusión de un tema que busca llegar a un punto que favorezca el insight de los pacientes o les permita identificar la necesidad de nuevas herramientas para su problemática, lo que después trabajan de manera individual con su psicoterapeuta, terapeuta ocupacional o psiquiatra.

\subsection{Relajación y Meditación}

Con el objetivo de manejar estrés y ansiedad en los pacientes, se practican diferentes técnicas de relajación. Para esta actividad, se tiene el resguardo de no incluir pacientes: psicóticos, maníacos o hipomaníacos, retraso mental moderado y severo, adictos intoxicados, los cuales podrían interferir en la relajación de los demás.

La meditación es una práctica que invita a la atención plena. Su función es proponer una paradoja, solicitando al paciente que combine un estado de serenidad con dejar de evadir aquello que produce dolor, prestarle atención total, abrirse a sentir esa experiencia, aceptarla sin juicios de valor e incorporarla a la vida.

Esta práctica facilita patrones más adecuados de regulación afectiva, permitiendo que la persona no sea dominada por estados mentales patológicos. Un ejemplo de estos estados mentales es el caso de cuadros depresivos, donde se presentan como parte de la sintomatología sentimientos de minusvalía y desesperanza que se instalan y se auto-perpetúan y es en la práctica de la meditación donde el paciente puede identificar estos pensamientos y elaborarlos adaptativamente.

\subsection{Psicoeducación}

El enfoque de la psicoeducación dada por TO está orientado a los siguintes objetivos: que los pacientes conozcan su patología y que desde este conocimiento logren una mejor adherencia al tratamiento, tengan herramientas para prevenir recaídas y logren reconocer signos y síntomas.

Por otro lado, hay charlas de psicoeducación orientadas a un mejor desempeño en el ámbito psicosocial. Por lo tanto la psicoeducación funciona como una propuesta para hacer cambios en la vida que conduzcan a una mejor calidad de vida, a mejorar las relaciones con la red de apoyo y mayor integración.

Mientras más informado esté el paciente y su red de apoyo es mejor la reinserción social y el manejo de la enfermedad, por lo tanto mejora el pronóstico y previene recaídas.

\subsection{Ergoterapia}

Esta actividad propia de la $\mathrm{T}$ O se realiza todos los días durante la tarde. El concepto de Ergoterapia deriva 
de los términos griegos: ergein = hacer, actuar, efectuar, trabajar; $y$ therapeios = servir, cuidar, curar. Por lo cual, se entiende como "la utilización del trabajo como medio de sanación". Sin embargo, tal como nos explican A. Guajardo y S. Zurita, no se trata de cualquier trabajo, sino de uno libre, personal, creativo y no alienante, donde lo importante no es el valor comercial del producto, sino el sentido que la actividad tiene para el paciente (Guajardo \& Zurita, 1991).

La actividad como medio de exprensión posee la finalidad de descubrir las habilidades latentes, redescubrir capacidades, lograr el auto conocimiento. Esto no significa que toda actividad sea terapéutica, ni que todo individuo que realice tareas para lograr satisfacer sus necesidades de expresión logre su "curación". La actividad en sí misma, no cura y no enferma; lo que es terapéutico es el método y la finalidad con la que se aplica para enriquecer a la persona; estructurando su personalidad, restableciendo los vínculos afectivos.

Podemos decir que el hombre tiene necesidades de expresar sus vivencias emocionales, sentimientos, utilizando con predominio el lenguaje estético y dinámico más que el verbal. La expresión estética pertenece al dominio de lo afectivo aunque su compresión exija un cierto nivel intelectual.

Creek identifica cuatro estrategias diferentes para aplicar actividades creativas como método de intervención:

- Como una forma de psicoterapia: utilizando la actividad artística como un medio, que permita al individuo explorar, elaborar y modificar sus pensamientos, emociones y comportamientos. Esto se fundamenta en el carácter proyectivo de las actividades artísticas desde un punto de vista psicodinámico.

- Como un vehículo para la expresión: que complementa o sustituye el lenguaje o la palabra.

- Como un juego, en el caso de la población infantojuvenil.

- Como agentes para la promoción de la salud y la prevención de la enfermedad y la discapacidad, en la medida que la actividad creativa fortalece la flexibilidad emocional (Creek, 2008).

Otro aspecto relevante es el "estado de flujo" que experimentan los pacientes durante estas sesiones. Entendiendo por "fluir" como una emoción positiva que consiste en quedar inmerso y absorto en una actividad, tanto, que se pierde la noción del tiempo y del mundo, según define Mihaly Csikszentmihaly. "Flow" se consigue en aquellas actividades en las que hay un equilibrio perfecto entre la habilidad y el gusto por realizar una actividad, la cual presenta al menos un pequeño desafío (Csikszentmihaly, 1997)

Dado lo anterior es que la ergoterapia es una intervención valorada y esperada por los pacientes, reportándolo como una de las instancias más beneficiosas y creativas de su tratamiento. Se realiza diariamente durante 2 horas en la tarde.

Para el desarrollo de la ergoterapia en esta unidad se ocupan materias primas que garanticen seguridad en el contexto de una hospitalización psiquiátrica aguda. Es por esto que la elección del material depende de los objetivos de tratamiento que plantee la terapeuta ocupacional a cargo, en conjunto con las motivaciones, intereses y capacidades del paciente. Teniendo en cuenta ambos puntos, la ergoterapia no sólo se enfila como una técnica que desarrolla la creatividad y la expresión de la persona, sino que también permite abarcar y mejorar diversas potencialidades neuropsicológicas y sociales.

En estas sesiones se utiliza una amplia variedad de artículos y materiales. Esta diversidad favorece el proceso creativo y de exploración.

Durante las sesiones de ergoterapia, se evalúa a los pacientes en el contexto social, habilidades cognitivas y en la organización o planificación de su trabajo. Es importante para el equipo poder objetivar el desempeño de los pacientes con las vulnerabilidades caracterológicas de cada uno, como por ejemplo: tolerancia a la frustración, impulsividad, respeto a normas o pares, entre otras.

\subsection{Actividad física}

Es un programa grupal, con objetivos en la estimulación motora y cognitiva, con un énfasis en lo primero.

Uno de los beneficios de estar insertos en un hospital general es contar con el apoyo multi-profesional de otros servicios, por lo cual hemos delegado la actividad física al equipo de kinesiólogos, quienes desarrollan dicha actividad en la sala de TO la que cuenta con el equipamiento necesario para realizarla adecuadamente y esto nos permite incluir a la mayoría de los pacientes. Se busca no sólo activar a los pacientes promoviendo salud a través de la actividad física, sino que además de contrarrestar efectos de psicofármacos o síntomas propios de las patologías que los aquejan. 
Las sesiones se realizan con una frecuencia de 2 veces por semana, con una duración de 45 minutos.

\subsection{Estimulación cognitiva/sensorial}

Actividades destinadas a prevenir, o rehabilitar las funciones mentales superiores: atención, memoria, concentración, orientación, percepción, cálculo matemático.

La aplicación específica de estimulación cognitiva en pacientes con adicción en periodo de desintoxicación tiene una función fundamental en el tratamiento, favoreciendo el insight del paciente (G. Rojo Mota, 2011), con ejercicios adecuados que aumenten la plasticidad cerebral y funciones cognitivas que permitan reflexionar hacia el cambio.

Además, estos principios se aplican en otras patologías, tales como: esquizofrenia, depresión, enfermedades degenerativas del sistema nervioso que evoluciona hacia la demencia, secuelas de accidentes vasculares.

Por otro lado, la estimulación sensorial busca aumentar canales sensitivos que han sido privados por el periodo de hospitalización y de la evolución de la patología.

\subsection{Actividades recreativas}

Dentro de las actividades de TO diseñadas para los diferentes ciclos de trabajo, se ha incorporado la recreación como una intervención fundamental dentro del tratamiento, dada una comprensión del juego como fenómeno cultural y no simplemente en sus aspectos biológicos, psicológicos o etnográficos, concibiéndolo como una función humana tan esencial como la reflexión y el trabajo. (Huizinga, 1972).

El juego se ha utilizado en nuestra disciplina desde sus inicios. En el adulto, la actividad lúdica puede ser utilizada para relajar, socializar y desarrollar destrezas, fisicas, cognitivas y sociales. El juego, el ocio y la recreación amplían los intereses, necesidades y capacidades de la fantasía. La realidad y la fantasía estan combinadas, fundidas con armonía para producir experiencias que no se encuentran fácilmente en las interacciones convencionales en el mundo real. (Romero D., 2003).

Desde la investigación evolutiva del juego, se observa que es una herramienta fundamental en la adaptabilidad de las especies, es un recurso de sobrevivencia, por lo cual se vuelve una necesidad inherente al ser humano, permitiendo el acceso a un entrenamiento natural de habilidades, con una mayor tasa de apre- hensión. La presencia del juego como estrategia de intervención permite por tanto que los pacientes de esta unidad logren integrarse socialmente. El objetivo de este programa de predominio lúdico es que el paciente interaccione con este recurso, logrando socializar, reducir el estrés de la hospitalización, explorar intereses, estimular elementos cognitivos, innovar en las respuestas mejorando adaptabilidad.

Lo terapéutico es usar el juego como un medio y no como un fin. (Brown, 2009).

Ayuda al paciente a asociar, reflexionar y elaborar sus conflictos, a reevaluar su autoconcepto, sus relaciones con la realidad exterior y y las relaciones interpersonales.

Trabajar la plasticidad de pensamientos y comportamientos al vivenciar diferentes roles, tolerar la frustración, integrarse e identificarse con el grupo, relacionarse desde diferentes canales de comunicación (Durante P., 2000).

Se desarrollan una vez por semana, dentro y fuera del espacio hospitalario.

En otras oportunidades, se traslada a los pacientes fuera del contexto hospitalario a diferentes dispositivos de la comunidad.

Esta es una actividad muy apreciada por los pacientes. Es importante señalar que sólo participan en las salidas aquellos que están voluntariamente hospitalizados (la hospitalización administrativa no es compatible), medianamente compensados, con autorización de su psiquiatra tratante.

Ayuda al paciente a asociar, reflexionar y elaborar sus conflictos, a reevaluar su autoconcepto, sus relaciones con la realidad exterior y y las relaciones interpersonales.

Trabajar la plasticidad de pensamientos y comportamientos al vivenciar diferentes roles, tolerar la frustración, integrarse e identificarse con el grupo, relacionarse desde diferentes canales de comunicación (Durante P., 2000).

\section{Trabajo Individual}

\subsection{Evaluación}

$\mathrm{Al}$ ingreso, los pacientes son discutidos en una reunión clínica con el equipo multidisciplinario. Tras la presentación del paciente, éste es integrado a las actividades de TO. Es en esta instancia donde inicialmente se observa al paciente en su integración, ánimo, conducta $y$ habilidades remanentes entre otras. 
Además, se le aplican según la necesidad, algunas de las siguientes evaluaciones:

- Evaluación de Roles (Romero Ayuso, 2001)

- Listado de Intereses (Matsutsuyu, J. revisado por Rogers, J.)

- Listado de roles (Oakley F.)

- Escala de calidad de vida

- Configuración individual de actividades (Cynkin, 1991; modificado por Romero Ayuso, 1998)

- Evaluación del hogar de T.O. (Romero y Ayuso, 2001)

- Inventario de intereses de ocupaciones de ocio "LOII" (Stein y Cutler, 1997)

- Escala de Lawton de actividades instrumentales de la vida diaria

- Evaluación de habilidades sociales

- MoCa.

Dentro de los métodos más utilizados de evaluación es la observación en la actividad como instrumento para objetivar comportamientos, capacidad funcional de las diferentes áreas ocupacionales. (Martijena, 1999).

Con frecuencia se utiliza la entrevista clínica como método de evaluación, con el propósito de conocer disfunciones ocupacionales en situación real, es decir, donde se desenvuelve habitualmente. Estas entrevistas son de tipo abierto, lo que permite tener la libertad para formular nuevas preguntas o intervenir en el momento.

\subsection{Evolución}

Durante la estadía de los pacientes, se mantiene la observación de ellos en su desenvolvimiento en la sala de TO lo que es reportado en ficha médica y discutido con cada tratante en reunión clínica.

\subsection{Intervención}

La intervención incluye la aplicación de métodos y técnicas adecuadas para lograr los objetivos propuestos, teniendo en cuenta el diagnóstico y caracteristicas individuales de la persona.

Con la interacción terapeuta ocupacional - paciente se intenta provocar cambios a través del aprendizaje, lo que permite que la persona pueda ver las consecuencias de sus acciones y establecer comportamientos ocupacionales satisfactorios.

Las actividades son muy variadas y dependen de los objetivos que se deseen alcanzar, de la motivación intrínsica de la persona y de sus intereses.
Se trabajan objetivos a corto plazo para el post alta. Para estas intervenciones, en algunos casos es necesario realizar visitas domiciliarias, visitas al centro de estudio o trabajo de cada paciente, para poder sugerir los cambios indicados para la reinserción de cada paciente.

Dentro de las estrategias utilizadas como intervención individual, se encuentra el acompañamiento terapéutico a los alrededores de la clínica con el fin de descomprimir el estrés de la internación, poder evaluar al paciente en un ambiente diferente, evaluar la socialización y manejo en la comunidad o para mejorar el vínculo.

En la preparación del alta y cuando es indicado, los pacientes quedan contactados y derivados a diferentes centros de intervención psico-social o a dispositivos comunitarios.

\subsection{Psicoeducación individual}

El alta genera ansiedad en los pacientes, por lo que es necesario orientación y educación para una vida independiente fuera de la clínica.

Por ello resulta conveniente que asuma gradualmente sus responsabilidades, desde lo relacionado con su autocuidado personal, la realización de lo quehaceres domésticos, la reanudación de la actividad académica o laboral, hasta todo aquello que concierne a su enfermedad y al tratamiento, con el fin de desarrollar estrategias que le permitan un mayor control, posibilitándole la prevención de nuevos episodios o recaídas." Las intervenciones psicoeducativas han llegado a ser parte fundamental de todo programa de reahabilitación y foco de intervención preferente en aquellos sujetos en los que se detecta un alto riesgo de recaídas" (Lukoff, Ventura, Nuechterlein y Liberman, 1992; Perry, 1995) (Rodríguez, 1997).

Existe un especial énfasis en la adherencia al tratamiento farmacológico y psicoterapéutico, ya que la mayor parte de los reingresos son por abandono del tratamiento.

En carta enviada a un diario local el Dr. R. Florenzano se refiere a un estudio realizado en el Servicio de Salud Metropolitano Oriente en colaboración con la Universidad del Desarrollo, encontrando que menos del $40 \%$ de los casos sigue tomando sus medicamentos y asistiendo a control al cabo de seis meses. Este es el principal obstáculo para el éxito del tratamiento y la experiencia coincide con los datos del estudio en los Estados Unidos y de la evaluación de la Escuela de Salud Pública de la Universidad de Chile. 
Mejorar la adherencia, se consigue con intervenciones psicosociales. La evidencia empírica actual señala que estas no son alternativas sino complementos a los psicofármacos. Las intervenciones psicoeducacionales mejoran los resultados incluso de las formas más refractarias (Florenzano, 2012).

\subsection{Planificación salidas terapéuticas}

Previo al alta, se realizan salidas terapéuticas a cargo de los familiares, donde los pacientes salen del ambiente hospitalario para ir a sus hogares durante un periodo de tiempo determinado, con el fin de constatar su re-integración en un ambiente normalizado, para luego ajustar el tratamiento a su realidad. Estas salidas terapéuticas son planificadas en conjunto con el paciente y el equipo de terapeutas, dado que es una situación de alto estrés tanto para ellos como para sus familiares.

El objetivo es estructurar el tiempo y las actividades a realizar promoviendo una reintegración saludable, manejo de imprevistos y proyección para el alta.

Luego de la salida terapéutica se evalúa la experiencia, rescatando los aspectos positivos y trabajando sobre los incidentes. Una vez resueltos estos aspectos se planifica la semana del paciente post alta, considerando que tendrá un control médico durante los próximos 7 días y una serie de indicaciones para su cuidado y manejo.

Esta planificación realizada por el equipo de terapeutas ocupacionales enmarca las actividades que el paciente escoge y significan un factor protector para él.

\subsection{Preparación para el alta}

En estas sesiones, en las que participan el paciente y/o la familia, se incluye ayuda y soporte para iniciar su tratamiento ambulatorio, estructuración del tiempo y programa de actividades durante la convalescencia, disponibilidad de recursos comunitarios, establecimiento de metas reales al retornar a su casa.

La información obtenida de la salida terapéutica es de mucha utilidad para proponer un plan de alta acorde a las expectativas y la realidad del paciente.

Durante dichas sesiones se establecen fechas de control por TO en caso de ser requerido.

Uso de computadores: En determinados casos, los pacientes pueden tener acceso a computadores e internet en la sala de TO bajo la supervisión de terapeutas ocupacionales y con la autorización del médico tratan- te. Existe un horario específico para su uso, dado que es una herramienta terapéutica para el equipo.

Con el uso del computador se busca la normalización del paciente, facilitar la comunicación con su medio externo, promover la socialización, permite un momento de distensión, de realización de trámites necesarios, investigan y aprenden sobre su enfermedad $u$ otros temas que le sean de su interés, los que en ocasiones exponen y comparten con sus compañeros de hospitalización, entre otros usos.

Se tiene la precaución de que el uso de internet sea personal, no se contacte con personas que no favorezcan el periodo de recuperación, que no se divulgue información potencialmente riesgosa o confidencial. El computador es de uso restringido para determinados pacientes.

\section{Familia}

\subsection{Psicoeducación}

Las familias tiene una función clave en el cuidado, y mantenimiento en la comunidad de su familiar enfermo.

La gran mayoría de nuestros pacientes viven con sus familias y ellas constituyen el principal recurso de atención, cuidado y soporte social. La problemática de la familia y sus necesidades es sub-atendida, lo que lleva a que muchas toleren las dificultades, tensiones y sobrecarga sin contar con la adecuada ayuda, apoyo y orientación. (Rodríguez, 1997).

La terapia familiar psicoeducativa es un modelo que fundamentalmente se inicia en el trabajo con familiares de personas con esquizofrenia. Aquí el objetivo es estabilizar al paciente, mientras que la familia debe aprender sobre síntomas, evolución, efectos de los fármacos.

Siguiendo este modelo es que se desarrollaron talleres para: trastorno bipolar, depresión, trastorno alimentario, esquizofrenia.

En la unidad se utiliza principalmente la educación familiar conductual, que consiste en formar y utilizar a diferentes miembros de la familia como administradores de respuestas adaptativas frente a contingencias, modificando al mismo tiempo modelos erróneos de funcionamiento en las relaciones familiares.

El objetivo es conseguir que las familias se vayan ajustando de modo flexible a los diferentes elementos que ocurren en los cambios, manteniendo una visión abierta al mundo. (Moruno P., 2012). 
Los cuidadores requieren atención a sus dificultades y problemas, que en muchos casos afrotan de un modo aislado sin apoyo ni orientación y vulnerados en sus derechos. Además, cuando se contiene a estos cuidadores, logramos una mejora en el vínculo con las familias, lo que favorece el intercambio de información relevante acerca del paciente.

Al finalizar cada sesión se les entrega material escrito de manera de complementar y reforzar lo expuesto, así como también se les sugiere bibliografía adecuada a las necesidades individuales de los familiares.

\section{Docencia}

Esta práctica se enmarca en un trabajo sistemático de docencia realizado desde el año 2003. El establecimiento como campo clínico de la Facultad de Medicina de la Universidad Católica de Chile donde los terapeutas ocupacionales de esta unidad participan en la formación de becados de psiquiatría y alumnos de medicina de $\mathrm{V}$ y VII año, promoviendo la visión y el trabajo de TO en salud mental.

Es así como becados de psiquiatría e internos de medicina deben vivenciar la experiencia de utilizar la ocupación como herramienta terapéutica bajo la supervisión y guía de los terapeutas ocupacionales de la unidad. Desde el año 2008, se desarrolla la labor de supervisores clíni- cos. La unidad recibe alumnos de $5^{\circ}$ año de la escuela de Terapia Ocupacional de la Universidad Austral de Chile, para realizar su práctica profesional durante 8 semanas.

\section{Resumen de la experiencia}

Durante estos 12 años hemos atendido 4.200 pacientes, siendo la prevalencia los trastornos del ánimo $47 \%$, las adicciones $18 \%$, esquizofernia y otras psicosis $9.1 \%$, trastornos alimentarios $6,1 \%$.

El programa de atención ha sido bien evaluado por los pacientes y ello se ve reflejado en la investigación, de una tesis doctoral realizada en el servicio (2010-12) con pacientes que ingresaron por intento de suicidio grave. En ella califican la percepción de la ayuda recibida. "Las ayudas calificadas como mayoritariamente beneficiosas son psiquiatría $84 \%$ terapia ocupacional $81 \%$, psicología 81\% enfermería 73\%." (Morales, 2012).

El $70 \%$ de los encuestados eran hombres y $30 \%$ mujeres.

En general destacan las actividades de terapia ocupacional y los espacios que pueden compartir con otros. Ellos valoran conversar con otros pacientes y consideran estas actividades "útiles" y las esperaban con entusiasmo cada dia".

Destacan el valor que le otorgan a las intervenciones individuales de las terapeutas. (Morales, 2012).

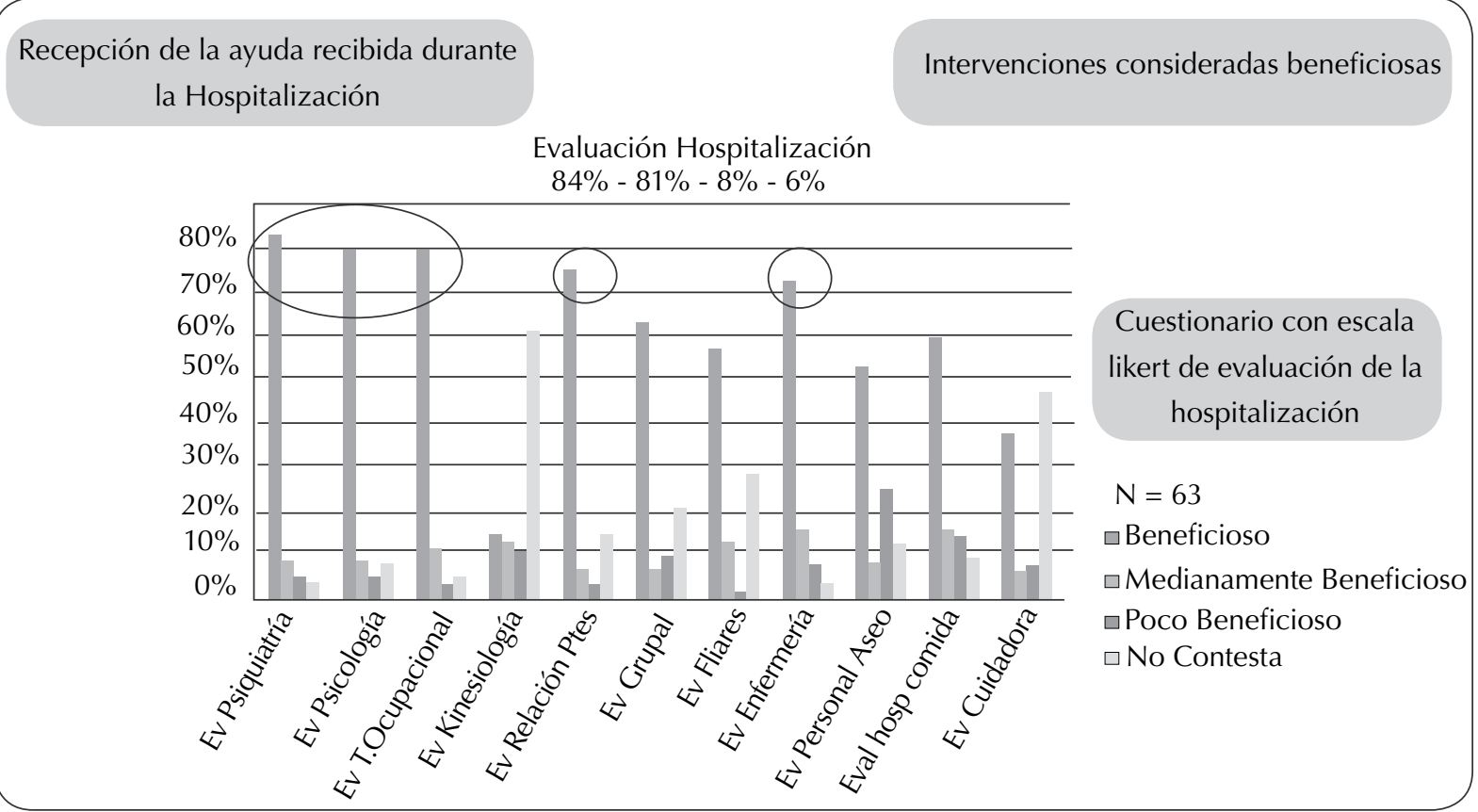




\begin{tabular}{|c|c|c|c|c|c|c|c|c|c|c|c|}
\hline & $\begin{array}{l}\text { Ev Psi- } \\
\text { quiatría }\end{array}$ & $\begin{array}{l}\text { Ev Psi- } \\
\text { cología }\end{array}$ & $\begin{array}{l}\text { Evalua- } \\
\text { ción T.O }\end{array}$ & $\begin{array}{l}\text { Ev Kine- } \\
\text { siología }\end{array}$ & $\begin{array}{l}\text { Ev Rela- } \\
\text { ción Ptes }\end{array}$ & $\begin{array}{c}\text { Ev Gru- } \\
\text { pal }\end{array}$ & $\begin{array}{c}\text { Ev } \\
\text { Fliares }\end{array}$ & $\begin{array}{l}\text { Ev En- } \\
\text { fermería }\end{array}$ & $\begin{array}{l}\text { Ev Perso- } \\
\text { nal Aseo }\end{array}$ & $\begin{array}{c}\text { Eval hosp } \\
\text { comida }\end{array}$ & $\begin{array}{l}\text { Ev Cui- } \\
\text { dadora }\end{array}$ \\
\hline Beneficioso & $84 \%$ & $81 \%$ & $81 \%$ & $14 \%$ & $76 \%$ & $63 \%$ & $57 \%$ & $73 \%$ & $54 \%$ & $60 \%$ & $38 \%$ \\
\hline $\begin{array}{c}\text { Medianamente } \\
\text { Beneficioso }\end{array}$ & $8 \%$ & $6 \%$ & $11 \%$ & $13 \%$ & $6 \%$ & $3 \%$ & $13 \%$ & $13 \%$ & $8 \%$ & $16 \%$ & $6 \%$ \\
\hline Poco Beneficioso & $5 \%$ & $5 \%$ & $3 \%$ & $11 \%$ & $3 \%$ & $10 \%$ & $2 \%$ & $8 \%$ & $25 \%$ & $14 \%$ & $8 \%$ \\
\hline No Contesta & $3 \%$ & $3 \%$ & $5 \%$ & $62 \%$ & $14 \%$ & $21 \%$ & $29 \%$ & $3 \%$ & $3 \%$ & $10 \%$ & $48 \%$ \\
\hline Totales & $100 \%$ & $100 \%$ & $100 \%$ & $100 \%$ & $100 \%$ & $100 \%$ & $100 \%$ & $100 \%$ & $100 \%$ & $100 \%$ & $100 \%$ \\
\hline
\end{tabular}

\section{CONCLUSIÓN}

En salud mental, la TO era entendida como una profesión para cuidar el ocio de los pacientes, disminuir los conflictos de los patios de recreo y cuidar de personas afectadas en su salud mental; se valorizó, principalmente tras el surgimiento de propuestas volcadas a la reinserción social y laboral, que propiciaban la horizontalidad de las relaciones entre el usuario, su familia, el equipo de salud y la comunidad, mejorando la calidad de vida y la dignidad de los pacientes.

En la medida que se ha evolucionado en la visión de la Salud Mental se ha desarrollado el tratamiento y la participación de una Terapia Ocupacional más compleja, precoz y sistemática, lo que implica un aporte económico significativo a la sociedad reflejado en una reinserción real de estos pacientes, menor tiempo de estadía en hospitalizaciones, mayor adherencia a tratamiento $y$ mejora en los pronósticos, disminuyendo cronicidad.

La ciencia de la ocupación ejerce un papel fundamental en la estructuración de conocimientos acerca de la ocupación, defieniendo aspectos que favorecen y promueven la participación e integración social de las personas, contribuyendo a involucrar a las personas en ocupaciones para dar soporte a la participación en su contexto y la mantención de su salud, promoviendo la participación cotidiana, por medio de sus ocupaciones.

Establecer programas como el rediseño de vida, son importantes por sus implicancias para la gestión de la salud, ya que la reorganización de las rutinas diarias pueden reducir la morbilidad y los efectos de la discapacidad (Araújo, Jordán, \& P. Gutiérrez Moclus, 2011).

Consideramos importante para nuestra disciplina dar a conocer esta experiencia dada la escasa literatura encontrada en relación a intervenciones en psiquiatría de patologías agudas en la práctica hospitalaria privada. En nuestro país tenemos una escasa oferta de estos dispositivos y la mayoria se encuentran en la ciudad de
Santiago. Es por esto que se vuelve necesario sistematizar el trabajo realizado para facilitar la replicación de programas que promuevan la integración y recuperación de pacientes psiquiátricos.

En este contexto, cabe señalar que en la experiencia de estos 12 años de servicio, el uso de la Ergoterapia como instrumento de intervención ha sido clave para el tratamiento de los pacientes, así como de reconocimiento de la importancia de la TO dentro del equipo médico. Hacemos esta reflexión dado que a pesar de contar con una larga tradición como método de intervención en TO, durante las últimas décadas la utilización de actividades artísticas y recreativas ha caído en desuso. Para muchos profesionales simboliza un descrédito para la profesión, quedando relegadas o rechazadas por los propios terapeutas ocupacionales como obsoletas, a pesar de que su uso se encuentra en el origen de nuestra disciplina. Es, por tanto, deber de nosotros el sistematizar y objetivar nuestro trabajo y logros, así como de educar y mostrar los beneficios de nuestro trabajo al equipo médico para acreditar la labor.

\section{REFERENCIAS BIBLIOGRÁFICAS}

Martijena, N. (1999). Terapia Ocupacional: Conceptos y aplicación. Mar del Plata: Martin.

Araújo, L. D.; Jordán, N. F. \& P. Gutiérrez Moclus, O. R. (2011). Ciencia de la Ocupación y terapia ocupacional: sus relaciones y aplicaciones a la práctica clínica. Revista Chilena de Terapia Ocupacional, 79-87.

Brown, S. (2009). Play. New York: Avery.

Creek, J. (2008). Occupational Therapy and Mental Health. Edimburg: Chrurchill Livingstone.

Cskszentmihaly, M. (1997). Finding Flow: the psychology of engagement with everyday life. New York: Basic Books. 
Durante P., N. B. (2000). Terapia Ocupacional en Salud Mental: 23 casos clínicos comentados. Barcelona: Masson.

Florenzano, R. (26 de abril de 2012). Pacientes con riesgo en Depresión. El Mercurio, pág. A2.

G. Rojo Mota, E. P. (2011). "Terapia Ocupacional en disfunción ejecutiva en drogodependencia". Revista de Neurología, 52: 163-72.

Guajardo, A. \& Zurita, S. (1991). Talleres de terapia ocupacional. Una experiencia con víctimas de la represión política. Santiago: Colección Cintras.

Huizinga, J. (1972). homo ludens. Madrid: alianza/emecé.

Moruno P, T. M. (2012). Terapia Ocupacional en Salud Mental. Barcelona: Masson.

Rodríguez, A. (1997). Rehabilitación Psicosocial de personas con trastornos mentales crónicos. $1^{\mathrm{a}}$ ed. Madrid: Pirámide.

Romero D., M. P. (2003). Terapia Ocupacional: teoría y técnicas. Barcelona: Masson.

Morales, S. (2012). "Significados y cambios asociados a la propia conducta suicida en pacientes hospitalizados por intento o ideacion suicida". Santiago: Tesis Doctoral, Doctorado en Psioterapia Pontificia Universidad Católica de Chile. 